\title{
Pulmonary complications associated with veno-arterial extra-corporeal membrane oxygenation: a comprehensive review
}

\author{
Aurélien Roumy ${ }^{1 *}$ D, Lucas Liaudet ${ }^{2}$, Marco Rusca ${ }^{2}$, Carlo Marcucci ${ }^{3}$ and Matthias Kirsch ${ }^{1}$
}

\begin{abstract}
Veno-arterial extracorporeal membrane oxygenation (VA-ECMO) is a life-saving technology that provides transient respiratory and circulatory support for patients with profound cardiogenic shock or refractory cardiac arrest. Among its potential complications, VA-ECMO may adversely affect lung function through various pathophysiological mechanisms. The interaction of blood components with the biomaterials of the extracorporeal membrane elicits a systemic inflammatory response which may increase pulmonary vascular permeability and promote the sequestration of polymorphonuclear neutrophils within the lung parenchyma. Also, VA-ECMO increases the afterload of the left ventricle (LV) through reverse flow within the thoracic aorta, resulting in increased LV filling pressure and pulmonary congestion. Furthermore, VA-ECMO may result in long-standing pulmonary hypoxia, due to partial shunting of the pulmonary circulation and to reduced pulsatile blood flow within the bronchial circulation. Ultimately, these different abnormalities may result in a state of persisting lung inflammation and fibrotic changes with concomitant functional impairment, which may compromise weaning from VA-ECMO and could possibly result in long-term lung dysfunction. This review presents the mechanisms of lung damage and dysfunction under VA-ECMO and discusses potential strategies to prevent and treat such alterations.
\end{abstract}

Keywords: Extracorporeal membrane oxygenation, Cardiogenic shock, Lung injury

\section{Introduction}

Veno-arterial extracorporeal membrane oxygenation (VA-ECMO) is a life-saving technology providing respiratory and circulatory support in patients with refractory cardiogenic shock or cardiac arrest [1] and which may give time to plan future therapeutic decisions such as the insertion of long-term cardiac assist devices or heart transplantation (HTX) [2]. Notwithstanding its potential benefits, VA-ECMO remains associated with significant morbidity and mortality [1]. This is partly due to the patients' critical condition, but also to complications related to VA-ECMO, notably renal failure, sepsis,

\footnotetext{
*Correspondence: aurelien.roumy@chuv.ch

'Department of Cardiovascular Surgery, University Hospital, Lausanne, Switzerland

Full list of author information is available at the end of the article
}

bleeding, thromboembolism, limb ischemia, and multiorgan failure $[3,4]$.

VA-ECMO-induced pulmonary complications are much less recognized, except from the pulmonary congestion related to left ventricle pressure overload induced by retrograde VA-ECMO flow within the thoracic aorta [5]. Beside this particular aspect, several additional mechanisms may contribute to lung damage and dysfunction in the setting of VA-ECMO. The latter may be assimilated to a simplified cardiopulmonary bypass $(\mathrm{CPB})$ circuit, and both techniques share common pitfalls with respect to lung physiology. $\mathrm{CPB}$ may alter pulmonary function after cardiac surgery by promoting an inflammatory response via biomaterial-dependent and biomaterial-independent factors, the collapse of lungs during the procedure, the shunting of pulmonary circulation, and the phenomenon 
of lung reperfusion injury which takes place once $\mathrm{CPB}$ is weaned [6]. During VA-ECMO, although such processes are attenuated, they still occur at different stages of support and at various degrees, and they may persist for days or weeks. In such conditions, the combination of a chronic inflammatory response, pulmonary congestion, and lung ischemia could foster a wealth of morphological and functional alterations which could interfere with patient's recovery and compromise the overall planned therapeutic strategy. In this review, we discuss the pathophysiological mechanisms and potential clinical implications of the pulmonary complications associated with VA-ECMO.

\section{VA-ECMO-related systemic inflammatory response syndrome}

The induction of a systemic inflammatory response syndrome (SIRS) by the contact of blood with biomaterial is a typical consequence of extracorporeal circulation [7].
While extensively studied in the field of $\mathrm{CPB}$, this is also witnessed during VA-ECMO, as recently reviewed [8]. In addition, patients undergoing VA-ECMO are critically ill and suffer from profound cardiogenic shock, which by themselves contribute to the development of SIRS [9]. The lung is a major target of inflammatory injury in the context of SIRS, owing to its extensive capillary bed and the presence of abundant immune cells within the lung parenchyma. Therefore, the occurrence of SIRS in the context of ECMO provides a highly favorable environment for the development of acute lung injury $[10,11]$. The main mechanisms triggering the inflammatory response to biomaterials are presented below.

\section{Humoral cascades}

Blood contact with VA-ECMO circuitry activates the contact system (CS) and the complement system (Fig. 1). CS generates kallikrein [12], which activates monocytes

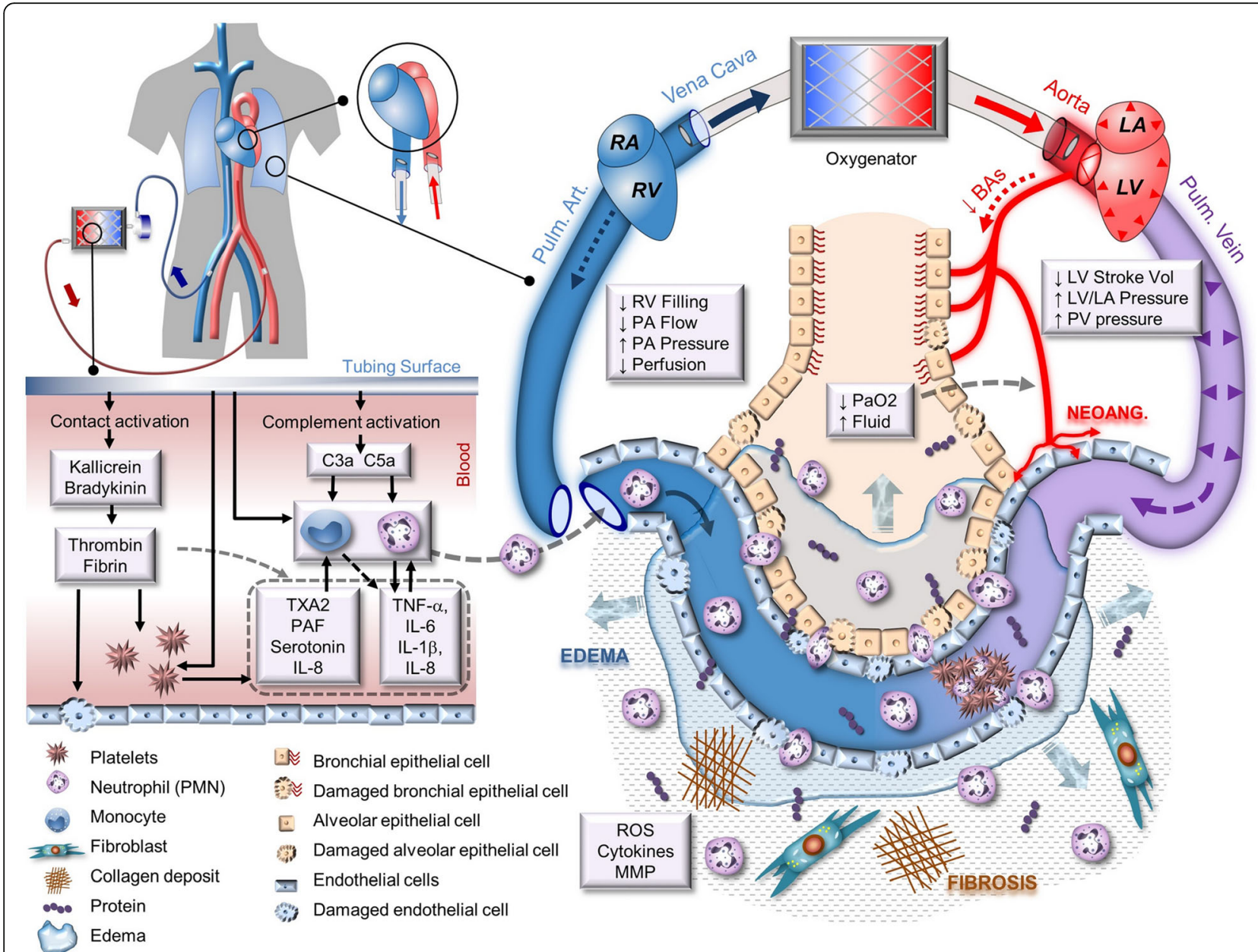

Fig. 1 Main VA-ECMO-induced mechanisms of lung damage and dysfunction. Left side: SIRS is initiated by the blood contact with the circuitry surface. It activates humoral cascades, platelets, and leukocytes, leading eventually to EC injury and activated PMN sequestration into the lung parenchyma. Right side: EC injury favors fluid infiltration into both alveolar space and lung parenchyma, leading to pulmonary edema, which is aggravated by the increase of pulmonary vein pressure. Alveolar edema and decreased pulmonary artery perfusion lead to lung parenchymal ischemia which in turn maintains chronic inflammation and promotes neoangiogenesis and fibrosis generation 
and polymorphonuclear cells (PMNs), and triggers the intrinsic coagulation cascade, resulting in the rapid generation of thrombin and fibrin within the systemic circulation [13]. Thrombin activates platelets and endothelial cells (ECs) and induces the secretion of pro-inflammatory mediators and growth factors, such as interleukin-6 (IL-6), interleukin-8 (IL-8), or platelet-derived growth factor (PDGF) [13]. The extrinsic coagulation pathway is activated to a lesser extent, mainly through the release of tissue factor (TF) by activated monocytes and ECs. CS also generates bradykinine [12], which activates ECs and leukocytes, and elicits hemodynamic alterations including systemic vasodilation and pulmonary vasoconstriction [14]. Complement activation occurs via the alternative pathway, generating the anaphylatoxins $\mathrm{C} 3 \mathrm{a}$ and $\mathrm{C} 5 \mathrm{a}$, which activate ECs. C5a is also a potent mediator of leukocyte chemotaxis. A peak of complement activation occurs within 1-2 h of ECMO onset, followed by a progressive decreases over the next 2 to 3 days [15].

The humoral response triggered by blood-biomaterial interaction comprises the release of multiple cytokines [16]. Whereas a balance between pro- and anti-inflammatory cytokines is reached several hours after VA-ECMO initiation [17], an initial imbalance in favor of pro-inflammatory TNF- $\alpha$, IL- $1 \beta$, and IL- 6 leads to the activation of ECs and promotes the release of multiple inflammatory proteins by the liver such as fibrinogen, complement, and C-reactive protein. TNF- $\alpha$ plays a major role in the amplification of the early inflammatory response, by upregulating proinflammatory cytokines and prostaglandin synthesis, activating PMNs and ECs, and stimulating reactive oxygen species (ROS) production $[18,19]$.

\section{Cell activation}

Platelets are activated by contact with the tubing surface and by thrombin and complement. Activated platelets foster the generation of pro-inflammatory cytokines, thromboxane A2 (TXA2), platelet-activating factor (PAF), Pselectin, and serotonin. TXA2 induces ECs activation and local vasoconstriction, while serotonin and P-selectin promote PMN-endothelial interactions [20]. Platelet activation is maximal at the initiation of VA-ECMO and progressively decreases over hours to days but remains persistent [21]. EC activation leads to their detachment from the basal membrane and disassembly of tight junctions, increasing vascular permeability with the development of sub-endothelial edema [22]. Moreover, activated ECs display an upregulated expression of adhesion molecules favoring PMN adhesion and transendothelial migration [23], and they also release cytokines, tissue factor, and ROS. Circulating PMNs, monocytes, and macrophages are spontaneously activated by tubing surfaces [24]. Furthermore, PMNs are activated by complement, histamine, serotonin, and PAF, which facilitate their adhesion to ECs, diapedesis, tissue infiltration [25], and the release of cytotoxic mediators, including proteases, cytokines, and ROS.

\section{Modulation of SIRS during VA-ECMO}

At variance with $\mathrm{CPB}$, VA-ECMO is generally maintained over several days. The initial significant SIRS gradually decreases $[15,17]$, mostly through the progressive build-up of counter-regulatory mechanisms leading to compensatory anti-inflammatory response and of possible biomaterial inactivation [26]. Still, a delayed persisting inflammatory response can be observed several days after VA-ECMO implementation, whose underlying mechanisms may involve the presence of low concentration of endotoxin within the circulation, which may sustain complement activation, cytokine release, and ROS generation, to elicit a sepsis-like inflammation [27, 28]. The low-level inflammatory response induced by pulmonary low flow is another potential mechanism (see below).

\section{Strategies to reduce SIRS-induced lung damages during VA-ECMO}

Some potential therapies have been proposed to downregulate inflammation and possibly improve lung outcome in this setting. The replacement of a silicon oxygenator by a poly-methyl pentene oxygenator has been associated with reduced radiological signs of pulmonary inflammation on chest X-ray [29], while the administration of steroids in patients undergoing VA-ECMO has been associated with shortened mechanical ventilation time, although without any survival benefit [30].

\section{VA-ECMO-related pulmonary congestion Pathophysiology}

Peripheral (femoro-femoral) VA-ECMO provides a non-physiological blood flow promoting significant hemodynamic perturbations (Fig. 1). The retrograde reinjection of blood into the thoracic aorta increases LV afterload and impedes aortic valve opening, while increasing myocardial oxygen demand [31]. In the setting of cardiogenic shock, these disturbances may worsen LV performance and dramatically reduce LV stroke volume [31, 32]. In addition, if LV residual function is insufficient to permit aortic valve opening, progressive LV distension will occur, due to persisting venous return through pulmonary and bronchial veins into the left atrium and through Thebesian veins into the LV, with concomitant increase of LV end-diastolic pressure. At worst, stagnation of blood within dilated left cardiac chambers may favor the formation of clots and induce pulmonary vein thrombosis [33].

Pulmonary congestion develops consecutively to the passive upstream transmission of elevated LV pressure [34]. Lung extravascular water accumulation is potentiated by the increased vascular permeability in the context of 
VA-ECMO-induced SIRS. The magnitude of afterload increase, LV distension, and pulmonary congestion is dependent on several parameters, including VA-ECMO flow, systemic vascular resistance, and LV residual function [31, 35].

Pulmonary congestion may jeopardize lung parenchymal cell oxygenation through two mechanisms. Firstly, interstitial edema increases the thickness of the alveolarcapillary barrier, hence the diffusion distance for oxygen between alveoli and parenchymal cells, whose oxygenation primarily depends on oxygen diffusing from alveolar spaces [36]. Secondly, alveolar edema results in a marked reduction of local alveolar $\mathrm{PO}_{2}\left(\mathrm{PAO}_{2}\right)$. Alveolar epithelial cells, normally exposed to $\mathrm{PAO}_{2}$ above 100 $\mathrm{mmHg}$, are sensitive to hypoxia from $\mathrm{PAO}_{2}$ below 50 $\mathrm{mmHg}$, which may occur in alveoli flooded by pulmonary edema [37]. Alveolar hypoxia can destabilize intercellular junctions, impair barrier permeability, impede alveolar fluid clearance and surfactant production by pneumocytes, induce local vasoconstriction and neoangiogenesis, and finally trigger local and systemic inflammation [37-39]. Therefore, pulmonary congestion during VA-ECMO creates a vicious circle in which VA-ECMOinduced SIRS and LV pressure overload promote pulmonary edema, leading to alveolar hypoxia which maintains SIRS [37]. Alveolar hemorrhages are another frequent consequence of the combination of pulmonary congestion and the requirement of anticoagulation during ECLS. Even if massive hemoptysis is rare [40], local alveolar hemorrhages are frequent and sustain local inflammatory changes $[41,42]$.

\section{Evaluation of pulmonary congestion and cardiac overload during VA-ECMO}

Chest X-ray is the simplest exam to assess pulmonary congestion, although its interpretation is complicated by frequently associated abnormalities, such as pneumonia, atelectasis, or alveolar hemorrhages. Chest ultrasound is an effective and reliable alternative method to assess interstitial edema, pleural effusion, and parenchymal consolidation [43]. Echocardiographic examination is mandatory, as it may show left heart dilation and indirect signs of cardiac congestion, such as spontaneous contrast echoes or the presence of "sludge" in heart chambers, as well as the absence of aortic valve opening [44]. Hemodynamic monitoring using pulmonary artery catheter (PAC) has been associated with improved survival in cardiogenic shock [45], notably in the context of mechanical cardiac support. PAC is particularly helpful to identify patients with cardiac distension, by demonstrating elevated left-sided filling pressure [46]. It has been shown that combining a value of pulmonary artery diastolic pressure $>25 \mathrm{mmHg}$ (as a surrogate of pulmonary capillary wedge pressure) with evidence of pulmonary edema on chest X-ray could identify patients with subclinical LV distension [47]. Although these data need further validation, PAC is now advocated by most experts to help manage patients under VAECMO [48].

\section{Strategies to reduce pulmonary congestion during VA- ECMO}

Severe pulmonary congestion during VA-ECMO is associated with a dismal prognosis, and its treatment is mandatory $[5,49]$. Inotropic agents increase cardiac contractility, promote aortic valve opening, and reduce LV dilation and filling pressure. Reducing VA-ECMO flow to decrease LV afterload, as long as residual LV ejection is present and peripheral perfusion maintained, should also be considered. The insertion of an intra-aortic balloon pump (IAPB) is a further option to decrease LV afterload. As demonstrated by Bréchot et al., IABP in combination with VA-ECMO versus VA-ECMO alone is independently associated with less frequent hydrostatic pulmonary edema and a shorter duration of mechanical ventilation [50]. A recent meta-analysis found concomitant IABP to reduce in-hospital death and length of stay [51].

If previous steps fail to reduce pulmonary edema, the left heart chambers must be directly unloaded ("vented"), either by percutaneous atrial transseptal approach or by using a venting cannula inserted into the left atrium or the LV apex by surgical or trans-aortic approach [52]. In addition, the catheter-mounted microaxial pump Impella ${ }^{\circ}$ (Abiomed, Danvers, MA) may represent a further efficient device to permit LV unloading [52]. Eliet et al. have recently observed that Impella ${ }^{\circ}$ not only decreases LV diastolic diameter but also increases pulmonary flow [53]. These different modalities of cardiac unloading during VA-ECMO have been the matter of several extensive recent reviews [52, 54].

\section{VA-ECMO-related lung ischemia}

\section{Lung blood supply}

The lung is characterized by a dual circulation, comprising the pulmonary circulation, which supplies the alveoli for gas exchange, and the bronchial circulation, which conveys oxygen and nutrients to the airways, but not alveoli, whose oxygen supply is almost exclusively provided by direct diffusion from the alveolar spaces [39]. Bronchopulmonary anastomoses allow collateralization between these two circulations. In case of chronic decrease of pulmonary blood flow (e.g., in chronic thromboembolic disease or pulmonary stenosis), the bronchial flow may increase from 1 to $30 \%$ of the cardiac output, permitting to compensate this decrease and participate to gas exchange, providing a kind of "rescue flow" to the ischemic areas $[55,56]$. 


\section{Disturbances induced by VA-ECMO}

As depicted in Fig. 1, venous blood during VA-ECMO is derived from the vena cava and the right atrium through the venous canula, resulting in a reduction of right ventricle (RV) filling, pulmonary blood flow, and pulmonary arterial pulsatility [31]. In a porcine model, Vardi et al. demonstrated that the pulmonary capillary blood flow decreases dramatically as the VA-ECMO flow increases [57]. Moreover, in case of pulmonary congestion (see above), the upstream transmission of increased left atrial pressure reduces the transpulmonary perfusion gradient. Ventilation with high positive end-expiratory pressure (PEEP) might also impede pulmonary blood flow by compression of alveolar vessels [58]. Several additional mechanisms, including alveolar hypoxia, reduction of local NO production, and the actions of inflammatory mediators can promote vasoconstriction and the subsequent increase of pulmonary vascular resistance, with a reduction of pulmonary blood flow [59]. It is also noteworthy that blood flow through the bronchial arteries (BAs) is also reduced during VA-ECMO, due to attenuated pulsatility of the systemic circulation (which supplies the BAs). This can further limit blood supply to ischemic areas within the congested lung [60]. Eventually, these various hemodynamic changes may lead to hypoperfusion of the entire pulmonary vasculature, which, superimposed to alveolar hypoxia, can promote a state of global, persistent lung ischemia.

\section{Strategies to reduce lung ischemia}

The best way to overcome such alterations is, of course, the withdrawal of VA-ECMO. If this is not possible, VAECMO flow may be reduced to maintain partial pulmonary perfusion. In early experimental studies in pigs, prolonged (18 h) ECMO at full support (with no residual pulmonary blood flow) promoted massive pulmonary parenchymal damage [61], which was not observed at a residual pulmonary blood flow reaching $25 \%$ of the systemic cardiac output [62]. An additional strategy relies in the upgrading of VA-ECMO to a hybrid system of veno-veno-arterial support, with an additional cannula inserted into the jugular vein, which provides oxygenated blood within the pulmonary arteries. This approach is sometimes used to treat the Harlequin syndrome (see below), but has not yet been evaluated to prevent lung injury during VA-ECMO.

\section{Structural lung parenchymal changes}

Although no dedicated study has specifically focused on pulmonary histological consequences of VA-ECMO, data from animal models and small human necropsy series have reported several pathological alterations. Koul et al. maintained 6 pigs under total CPB for $18 \mathrm{~h}$ before weaning. All the animals died within the next $4 \mathrm{~h}$, and on histological examination, more than $80 \%$ of the pulmonary parenchyma displayed edema, hyaline membranes, alveolar hemorrhages, thrombi, and focal necrotic changes [61]. In another experimental study exploring the effects of long-term VA-ECMO without anticoagulation, Mizuno et al. succeeded to maintain a goat up to 5 months under VA-ECMO with a pulmonary blood flow reduced to $40 \%$. At autopsy, diffuse interstitial fibrosis and swelling of endothelial cells with thickening of their basal membrane were noted [63].

In humans, Ratliff et al. reported postmortem findings in 4 patients undergoing VA-ECMO for 7 to 12 days. In two patients, diffuse lung fibrosis was noted, together with liquefaction necrosis of the lower lobes. The authors hypothesized that the combination of an increase in metabolically active cell mass together with partial pulmonary shunting concurred to establish ischemic areas with subsequent necrosis [64]. In an autopsy series of 23 infants supported by VA-ECMO, Chou et al. reported hyaline membrane formation, interstitial and intra-alveolar hemorrhages, and reactive hyperplasia of epithelial and smooth muscle cells, developing already after 2 to 3 days of VA-ECMO support, whereas interstitial fibrosis was noted beyond 7 days [41].

To sum up, VA-ECMO appears mostly associated with signs of protein-rich edema, alveolar hemorrhages, tissue necrosis, and fibrosis, which are reminiscent of the damage noted in the acute respiratory distress syndrome. These changes are likely the result of the combination of inflammatory injury, pulmonary congestion, and hypoxia, with the progressive development of epithelialendothelial injury, increased vascular permeability, and interstitial collagen deposition [65]. Furthermore, some degree of angiogenesis and vascular remodeling may also play some role, as alveolar hypoxia and chronic ischemia (typical of long-lasting VA-ECMO) can activate several pro-angiogenic cascades in alveolar cells, relying on the hypoxia-inducible factor family or the resistin-like molecule- $\alpha$ [37]. Such alterations could result in longterm changes in pulmonary vascular physiology, with possible detrimental consequences on the right ventricle.

\section{Potential clinical consequences}

\section{Pulmonary dysfunction during VA-ECMO}

The impaired pulmonary function induced by VA-ECMO may require long-lasting mechanical ventilation (MV) which may further alter the lung through ventilatorinduced lung injury (VILI). Although there is presently no consensus regarding optimal ventilator settings for $\mathrm{MV}$ during VA-ECMO, the principles of lung-protective ventilation should be applied [66].

Furthermore, prolonged MV increases the risk of ventilator-associated pneumonia (VAP), which occurs in up to $74 \%$ of patients under ECMO, as recently reviewed 
[67], with risk factors including an age $>65$ years, a higher SOFA score on admission, and a history of COPD or hypertension [68]. Causative microorganisms comprise primarily Gram-negative bacilli, with Pseudomonas aeruginosa isolated in 18-25\% of cases [67]. Diagnosis of VAP may be particularly troublesome, as the usual criteria of VAP are difficult to interpret in the setting of ECMO, and a high clinical index of suspicion coupled to early microbiological sampling are major clues to diagnosis [67]. Treatment of VAP on ECMO is challenging, notably because of the alterations of antibiotic pharmacokinetics occurring in this setting, and frequent therapeutic drug monitoring is therefore recommended [69].

Preventive measures to reduce the risk of VAP include primarily the reduction of MV duration. In this regard, a strategy of early extubation and awake ECMO support is emerging as a promising strategy [70]. In properly selected patients, such strategy not only significantly reduces the incidence of VAP [70], but also permits active mobilization, reduces the overall rate of complications, and increases survival [71].

The prototypical consequence of VA-ECMOdependent impairment of lung function is the development of the "Harlequin syndrome," reflecting the opposing flows from the heart (antegrade, poorly oxygenated blood flow) and from the peripheral ECMO (retrograde, highly oxygenated blood flow), resulting in differential hypoxia (upper body hypoxemia, lower body normo/ hyperoxemia). The level of mixing of the two flows within the aorta is termed the "watershed," which can be identified in contrast-enhanced CT scan of the chest (Fig. 2). The Harlequin syndrome may be treated by increasing VA-ECMO flow or adding a venous injection cannula either as a hybrid ECMO (veno-veno-arterial ECMO, Fig. 3) or as pure veno-venous ECMO if the function of the heart allows withdrawal of the arterial cannula. Another option consists of switching the arterial cannulation site from femoral to axillary or central (aorta) location, in order to avoid the retrograde flow from the peripheral femoral cannula [72].

Finally, bronchoscopy with bronchial hygiene may be considered routinely in order to maximize chances of successful weaning from VA-ECMO when cardiac function recovers [73].

\section{Pulmonary dysfunction after weaning from VA-ECMO}

VA-ECMO-induced lung alterations may only appear after weaning and the restoration of physiological pulmonary artery blood flow. In a series of 55 patients who underwent long-term mechanical assist device implantation under VA-ECMO (L-VAD, bi-VAD, or total artificial heart), Boulate et al. noticed that $27 \%$ of patients develop acute lung injury (ALI) few hours after restoration of pulmonary blood flow, with a significant impact on mortality. The authors hypothesized that chronic lung ischemia during VA-ECMO support could promote alveolar frailty and that the sudden restoration of an antegrade pulsatile pulmonary blood flow creates a massive pulmonary bed overload responsible of ALI [74]. Accordingly, one of the identified risk factors of ALI in this study was the occurrence of a pulmonary edema during the week preceding the implantation of the mechanical device, featuring a preexisting lung frailty.

This form of ALI is reminiscent of reperfusion pulmonary edema, a well-known and described condition that occurs after reperfusion of a chronic low pulmonary blood flow situation, as in correction of tetralogy of Fallot [75] or pulmonary endarteriectomy for chronic embolism [76]. Such reperfusion pulmonary edema relies both on ischemia-induced chronic inflammation and reperfusion injury that involves similar mechanisms than those described above [77].

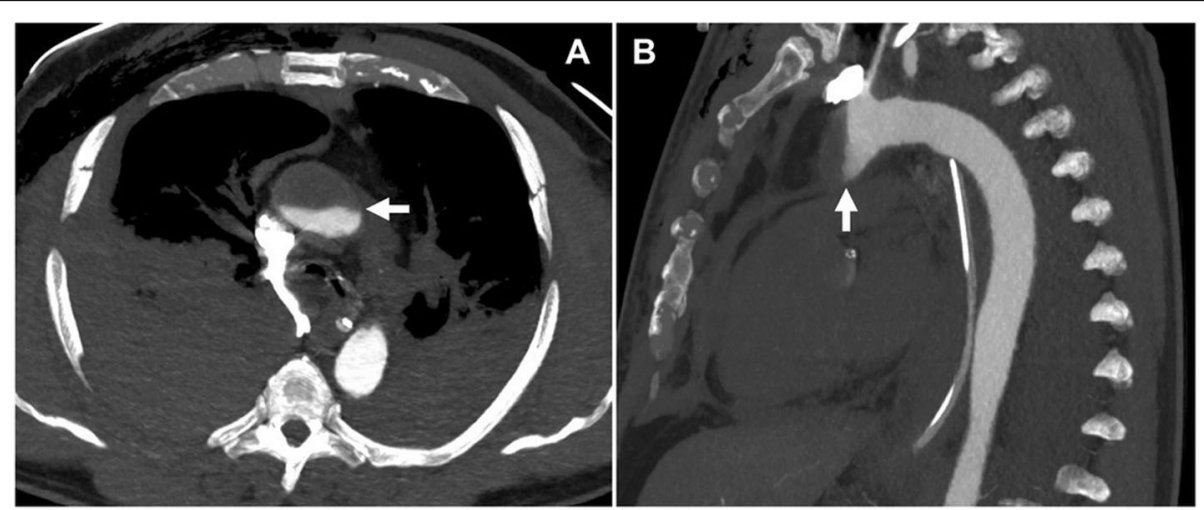

Fig. 2 The watershed. a Axial. b Sagittal. Contrast in the aorta indicates blood flow from the VA-ECMO arterial cannula, whereas absence of contrast within the ascending aorta indicates blood flow from the native heart. The level of blood mixing in the thoracic aorta represents the VAECMO watershed (arrows) 


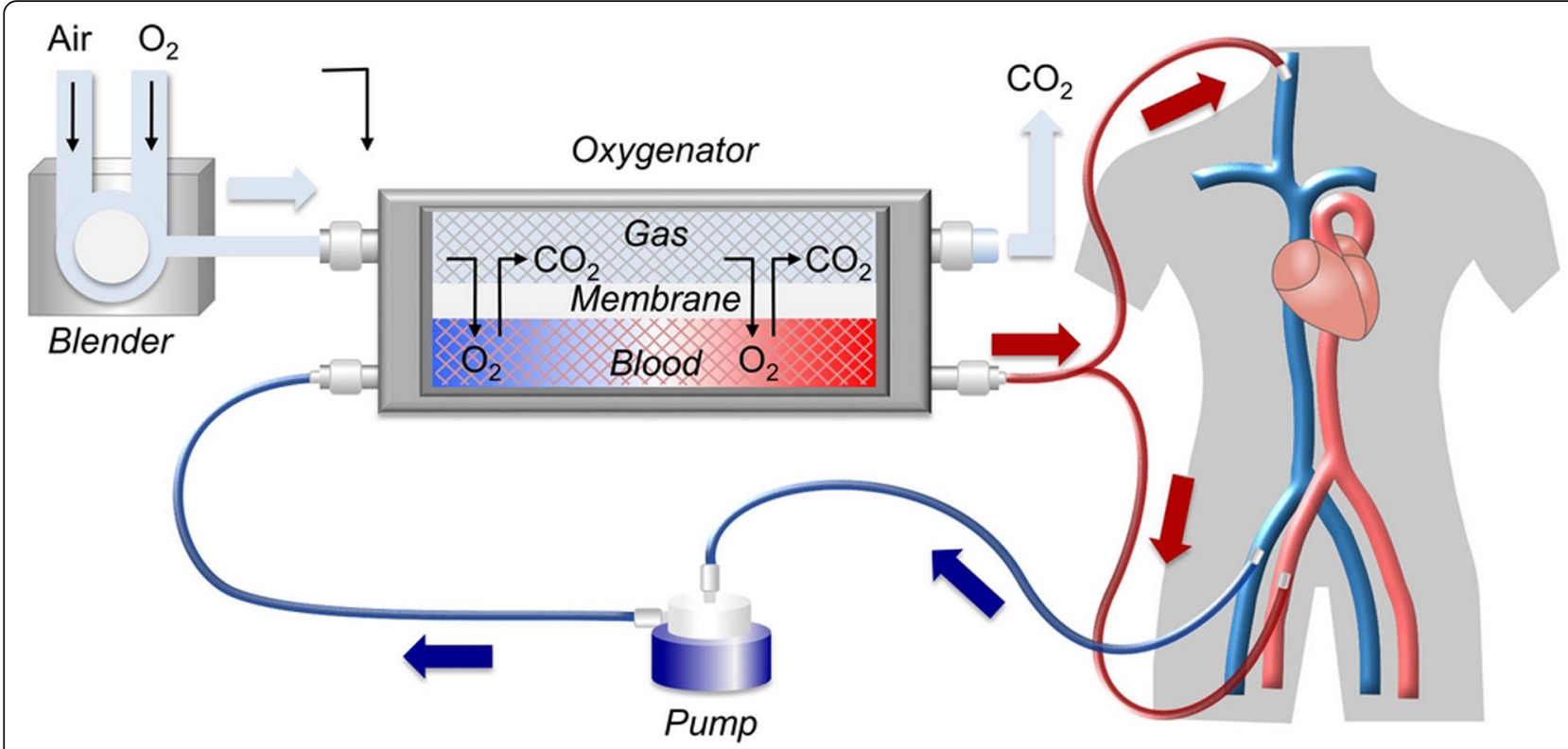

Fig. 3 Veno-veno-arterial ECMO. Oxygenated blood is propelled through both the femoral arterial cannula and the additional jugular cannula providing oxygenated blood directly into the right-heart chambers and consequently into the left atrium. This setting permits to wean progressively the arterial cannula in order to switch to veno-venous ECMO

In order to help the decision-making process in patients under VA-ECMO, Chen et al. developed a risk factorcalling score (RFSS) to select patients eligible for L-VAD or HTX. The RFSS has 5 items and 16 points, 7 of which are allocated to pulmonary dysfunction. A RFSS $>7$ predicted a poor outcome, which emphasizes the relative burden of pulmonary dysfunction in the outcome of patients under VA-ECMO in a bridge strategy [78].

\section{Long-term outcome}

It is currently unknown whether lung damage and dysfunction induced by VA-ECMO have an impact on long-term outcome, the more so that many unrelated factors may interfere with such outcome, such as prolonged ICU stay, previous health condition, or reduced LV ejection fraction. A few studies focused on long-term health-related quality of life (HRQL) in VA-ECMO survivors after cardiogenic shock. Combes et al. questioned 28 VA-ECMO survivors about their HRQL via the short-form 36 questionnaire (SF-36). Mean VA-ECMO duration and follow-up were respectively 7 days (5 to 10) and 11 months (3 to 39). In comparison to sex- and agematched controls, VA-ECMO survivors disclosed significantly lower role-physical score and a trend to a lower physical function, even though most patients recovered a good cardiac function with mean LVEF $51 \%$ or underwent HTX [79]. These results were confirmed by other studies showing lower SF-36 values of physical functioning and role-physical scores in VA-ECMO survivors compared to standard population $[80,81]$. These data however do not give any information with respect to the potential long-term burden of pulmonary alterations associated with VA-ECMO, and future studies should be designed to address this issue, for example by performing delayed lung functional tests in long-term survivors of VA-ECMO.

\section{Conclusion}

VA-ECMO elicits several pathophysiological disturbances which may significantly impact on lung integrity and function. First, the rapid development of a systemic inflammatory response with pulmonary involvement is an unavoidable consequence of the artificial VA-ECMO circuitry. Second, due to retrograde blood flow within the thoracic aorta, peripheral VA-ECMO has the propensity to increase LV afterload, which may favor the congestion of alveoli already affected by the ongoing inflammation.

Third, persistent lung ischemia due to the partial shunting of the pulmonary circulation and reduced pulsatility of the bronchial circulation may elicit further cytotoxicity within the whole lung parenchyma. Limited evidence from human observational studies and animal models indicates that VA-ECMO support for more than a few days may lead to severe structural changes of the lung parenchyma and interstitial fibrosis, which could result in long-term functional limitation. Clinicians in charge of VA-ECMO patients should be aware of its effects on lung physiology and should take all measures to limit such consequences, including the maintenance of 
VA-ECMO support as short as possible, the early diagnosis and treatment of cardiac overload and pulmonary congestion, and the application of lungprotective ventilation. Future studies specifically addressing the issue of the pulmonary consequences of VA-ECMO are warranted.

\section{Abbreviations}

ALI: Acute lung injury; ARDS: Acute respiratory distress syndrome; CARS: Compensatory anti-inflammatory response syndrome; CPB: Cardiopulmonary bypass; CS: Contact system; EC: Endothelial cell; HTX: Heart transplantation; IABP: Intra-aortic balloon pump; ICU: Intensive care unit; IL: Interleukine; LA: Left atrium; LV: Left ventricle; MMP: Matrix metalloproteinase; MI: Myocardial infarction; MV: Mechanical ventilation; PA: Pulmonary artery; PAF: Platelet-activating factor; PEEP: Positive end expiratory pressure; PMN: Polymorphonuclear; PV: Pulmonary vein; RA: Right atrium; ROS: Reactive oxygen species; RV: Right ventricle; SIRS: Systemic inflammatory response syndrome; TF: Tissue factor; TNF: Tumor necrosis factor; TXA: Thromboxane; VA-ECMO: Veno-arterial extracorporeal membrane oxygenation; VAD: Ventricular assist device; VAP: Ventilator-associated pneumonia; VILI: Ventilator-induced lung injury

\section{Acknowledgements}

Not applicable.

\section{Authors' contributions}

AR and MK designed and conceived the paper. AR wrote the manuscript. LL provided figures and was a major contributor in writing manuscript. All authors contributed to the content of this paper and critically reviewed the final manuscript. AR edited individual contributions and finalized the manuscript. All authors read and approved the final manuscript.

\section{Funding}

Lucas Liaudet is supported by a grant from the Swiss National Fund for Scientific Research (Nr 310030_162629).

\section{Availability of data and materials}

Not applicable.

\section{Ethics approval and consent to participate}

Not applicable.

\section{Consent for publication}

Not applicable.

\section{Competing interests}

The authors declare they have no competing interests.

\section{Author details}

'Department of Cardiovascular Surgery, University Hospital, Lausanne, Switzerland. ${ }^{2}$ Department of Intensive Care Medicine, University Hospital, Lausanne, Switzerland. ${ }^{3}$ Department of Anesthesiology, University Hospital, Lausanne, Switzerland.

Received: 14 December 2019 Accepted: 30 April 2020

Published online: 11 May 2020

\section{References}

1. Ouweneel DM, Schotborgh JV, Limpens J, Sjauw KD, Engström AE, Lagrand WK, et al. Extracorporeal life support during cardiac arrest and cardiogenic shock: a systematic review and meta-analysis. Intensive Care Med. 2016; 42(12):1922-34.

2. Rousse N, Juthier F, Pinçon C, Hysi I, Banfi C, Robin E, et al. ECMO as a bridge to decision: recovery, VAD, or heart transplantation? Int J Cardiol. 2015;187:620-7.

3. Lafç $G, B$ Budak $A B$, Yener AÜ, OF C. Use of extracorporeal membrane oxygenation in adults. Heart Lung Circ. 2014;23(1):10-23.

4. Zangrillo A, Landoni G, Biondi-Zoccai G, Greco M, Greco T, Frati G, et al. A meta-analysis of complications and mortality of extracorporeal membrane oxygenation. Crit Care Resusc. 2013;15(3):172-8.
5. Guglin M, Burchett A, Tribble T, Charnigo R. Pulmonary congestion (white lungs) on VA ECMO. The VAD J. 2016;2(4).

6. Huffmyer JL, Groves DS. Pulmonary complications of cardiopulmonary bypass. Best Pract Res Clin Anaesthesiol. 2015;29(2):163-75.

7. Kraft F, Schmidt C, Van Aken H, Zarbock A. Inflammatory response and extracorporeal circulation. Best Pract Res Clin Anaesthesiol. 2015;29(2):11323.

8. Millar JE, Fanning JP, McDonald Cl, McAuley DF, Fraser JF. The inflammatory response to extracorporeal membrane oxygenation (ECMO): a review of the pathophysiology. Crit Care Lond Engl. 2016;20(1):387.

9. Cooper HA, Panza JA. Cardiogenic shock. Cardiol Clin. 2013;31(4):567-80 viii.

10. Luo S, Wang Y, An Q, Chen H, Zhao J, Zhang J, et al. Platelets protect lung from injury induced by systemic inflammatory response. Sci Rep. 2017;7: 42080.

11. Bhatia M, Zemans RL, Jeyaseelan S. Role of chemokines in the pathogenesis of acute lung injury. Am J Respir Cell Mol Biol. 2012;46(5):566-72.

12. Campbell DJ, Dixon B, Kladis A, Kemme M, Santamaria JD. Activation of the kallikrein-kinin system by cardiopulmonary bypass in humans. Am J Physiol Regul Integr Comp Physiol. 2001;281(4):R1059-70.

13. Chandler WL, Velan T. Estimating the rate of thrombin and fibrin generation in vivo during cardiopulmonary bypass. Blood. 2003;101(11):4355-62.

14. Golias C, Charalabopoulos A, Stagikas D, Charalabopoulos K, Batistatou A. The kinin system--bradykinin: biological effects and clinical implications. Multiple role of the kinin system--bradykinin. Hippokratia. 2007;11(3):124-8.

15. Graulich J, Sonntag J, Marcinkowski M, Bauer K, Kössel H, Bührer C, et al. Complement activation by in vivo neonatal and in vitro extracorporeal membrane oxygenation. Mediat Inflamm. 2002;11(2):69-73.

16. MclLwain RB, Timpa JG, Kurundkar AR, Holt DW, Kelly DR, Hartman YE, et al. Plasma concentrations of inflammatory cytokines rise rapidly during ECMOrelated SIRS due to the release of preformed stores in the intestine. Lab Invest. 2010;90(1):128-39.

17. Chung JH, Yeo HJ, Kim D, Lee SM, Han J, Kim M, et al. Changes in the levels of beta-thromboglobulin and inflammatory mediators during extracorporeal membrane oxygenation support. Int J Artif Organs. 2017;40(10):575-80.

18. Zhang $H$, Park $Y$, Wu J, ping $C X$, Lee $S$, Yang J, et al. Role of TNF-alpha in vascular dysfunction. Clin Sci (Lond). 2009;116(3):219-30.

19. Shi J, Chen Q, Yu W, Shen J, Gong J, He C, et al. Continuous renal replacement therapy reduces the systemic and pulmonary inflammation induced by venovenous extracorporeal membrane oxygenation in a porcine model. Artif Organs. 2014;38(3):215-23.

20. Thomas MR, Storey RF. The role of platelets in inflammation. Thromb Haemost. 2015;114(3):449-58.

21. Cheung PY, Sawicki G, Salas E, Etches PC, Schulz R, Radomski MW. The mechanisms of platelet dysfunction during extracorporeal membrane oxygenation in critically ill neonates. Crit Care Med. 2000;28(7):2584-90.

22. Ait-Oufella H, Maury E, Lehoux S, Guidet B, Offenstadt G. The endothelium: physiological functions and role in microcirculatory failure during severe sepsis. Intensive Care Med. 2010;36(8):1286-98.

23. Mittal M, Siddiqui MR, Tran K, Reddy SP, Malik AB. Reactive oxygen species in inflammation and tissue injury. Antioxid Redox Signal. 2014;20(7):1126-67.

24. Stefano GB, Bilfinger TV. Human neutrophil and macrophage chemokinesis induced by cardiopulmonary bypass: loss of DAME and IL-1 chemotaxis. J Neuroimmunol. 1993:47(2):189-97.

25. Graulich J, Walzog B, Marcinkowski M, Bauer K, Kössel H, Fuhrmann G, et al. Leukocyte and endothelial activation in a laboratory model of extracorporeal membrane oxygenation (ECMO). Pediatr Res. 2000;48(5):679-84.

26. Adib-Conquy M, Cavaillon J-M. Compensatory anti-inflammatory response syndrome. Thromb Haemost. 2009;101(1):36-47.

27. Boyle EM, Pohlman TH, Johnson MC, Verrier ED. Endothelial cell injury in cardiovascular surgery: the systemic inflammatory response. Ann Thorac Surg. 1997;63(1):277-84.

28. Hirthler M, Simoni J, Dickson M. Elevated levels of endotoxin, oxygenderived free radicals, and cytokines during extracorporeal membrane oxygenation. J Pediatr Surg. 1992;27(9):1199-202.

29. Khoshbin E, Dux AEW, Killer H, Sosnowski AW, Firmin RK, Peek GJ. A comparison of radiographic signs of pulmonary inflammation during ECMO between silicon and poly-methyl pentene oxygenators. Perfusion. 2007; 22(1):15-21

30. Westfall SH, Stephens C, Kesler K, Connors RH, Tracy TF, Weber TR. Complement activation during prolonged extracorporeal membrane oxygenation. Surgery. 1991;110(5):887-91. 
31. Lim HS, Howell N, Ranasinghe A. Extracorporeal life support: physiological concepts and clinical outcomes. J Card Fail. 2017;23(2):181-96.

32. Ostadal $P$, Mlcek M, Kruger A, Hala P, Lacko S, Mates M, et al. Increasing venoarterial extracorporeal membrane oxygenation flow negatively affects left ventricular performance in a porcine model of cardiogenic shock. J Transl Med. 2015;13:266.

33. Williams B, Bernstein W. Review of Venoarterial extracorporeal membrane oxygenation and development of intracardiac thrombosis in adult cardiothoracic patients. J Extra Corpor Technol. 2016;48(4):162-7.

34. Murray JF. Pulmonary edema: pathophysiology and diagnosis. Int J Tuberc Lung Dis. 2011;15(2):155-60 i.

35. Burkhoff D, Sayer G, Doshi D, Uriel N. Hemodynamics of mechanical circulatory support. J Am Coll Cardiol. 2015;66(23):2663-74.

36. Driehuys B, Cofer GP, Pollaro J, Mackel JB, Hedlund LW, Johnson GA Imaging alveolar-capillary gas transfer using hyperpolarized 129Xe MRI. Proc Natl Acad Sci U S A. 2006;103(48):18278-83.

37. Fröhlich S, Boylan J, McLoughlin P. Hypoxia-induced inflammation in the lung: a potential therapeutic target in acute lung injury? Am J Respir Cell Mol Biol. 2013;48(3):271-9.

38. Chen T, Yang C, Li M, Tan X. Alveolar hypoxia-induced pulmonary inflammation: from local initiation to secondary promotion by activated systemic inflammation. J Vasc Res. 2016;53(5-6):317-29.

39. Jain M, Sznajder Jl. Effects of hypoxia on the alveolar epithelium. Proc Am Thorac Soc. 2005;2(3):202-5.

40. Pitcher HT, Harrison MA, Shaw C, Cowan SW, Hirose H, Cavarocchi N. Management considerations of massive hemoptysis while on extracorporeal membrane oxygenation. Perfusion. 2016;31(8):653-8.

41. Chou P, Blei ED, Shen-Schwarz S, Gonzalez-Crussi F, Reynolds M. Pulmonary changes following extracorporeal membrane oxygenation: autopsy study of 23 cases. Hum Pathol. 1993;24(4):405-12.

42. Lee HE, Yi ES, Rabatin JT, Bohman JK, Roden AC. Histopathologic findings in lungs of patients treated with extracorporeal membrane oxygenation. Chest. 2018;153(4):825-33.

43. Pasero D, Persico P, Tenaglia T, Ranieri VM. Respiratory monitoring during VA ECMO. In: Sangalli F, Patroniti N, Pesenti A, editors. ECMO-Extracorporeal Life Support in Adults. Milano: Springer Milan; 2014. p. 383-8. Available from: http://link.springer.com/10.1007/978-88-470-5427-1_33. [cited 2019 May 7].

44. Eckhauser AW, Jones C, Witte MK, Puchalski MD. Transthoracic echocardiographic predictors of left atrial hypertension in patients on venoarterial extracorporeal membrane oxygenation. World J Pediatr Congenit Heart Surg. 2014;5(1):67-9.

45. Hernandez GA, Lemor A, Blumer V, Rueda CA, Zalawadiya S, Stevenson LW, et al. Trends in utilization and outcomes of pulmonary artery catheterization in heart failure with and without cardiogenic shock. J Card Fail. 2019;25(5): 364-71.

46. Krishnan S, Schmidt GA. Hemodynamic monitoring in the extracorporeal membrane oxygenation patient. Curr Opin Crit Care. 2019;25(3):285-91.

47. Truby LK, Takeda K, Mauro C, Yuzefpolskaya M, Garan AR, Kirtane AJ, et al. Incidence and implications of left ventricular distention during venoarterial extracorporeal membrane oxygenation support. ASAIO J. 2017;63(3):257-65.

48. Rao P, Khalpey Z, Smith R, Burkhoff D, Kociol RD. Venoarterial extracorporeal membrane oxygenation for cardiogenic shock and cardiac arrest. Circ Heart Fail. 2018;11(9):e004905.

49. Jamadar DA, Kazerooni EA, Cascade PN, Fazzalari FL, Vydareny KH, Bartlett RH. Extracorporeal membrane oxygenation in adults: radiographic findings and correlation of lung opacity with patient mortality. Radiology. 1996;198(3):693-8.

50. Thiele H, Zeymer U, Neumann F-J, Ferenc M, Olbrich H-G, Hausleiter J, et al. Intra-aortic balloon counterpulsation in acute myocardial infarction complicated by cardiogenic shock (IABP-SHOCK II): final 12 month results of a randomised, open-label trial. Lancet Lond Engl. 2013;382(9905):1638-45.

51. Li Y, Yan S, Gao S, Liu M, Lou S, Liu G, et al. Effect of an intra-aortic balloon pump with venoarterial extracorporeal membrane oxygenation on mortality of patients with cardiogenic shock: a systematic review and meta-analysis. Eur J Cardio-Thorac Surg. 2019;55(3):395-404.

52. Rupprecht L, Flörchinger B, Schopka S, Schmid C, Philipp A, Lunz D, et al. Cardiac decompression on extracorporeal life support: a review and discussion of the literature. ASAIO J. 2013;59(6):547-53.

53. Eliet J, Gaudard P, Zeroual N, Rouvière P, Albat B, Mourad M, et al. Effect of impella during veno-arterial extracorporeal membrane oxygenation on pulmonary artery flow as assessed by end-tidal carbon dioxide. ASAIO J. 2018;64(4):502-7.
54. Meani P, Gelsomino S, Natour E, Johnson DM, Rocca H-PBL, Pappalardo F, et al. Modalities and effects of left ventricle unloading on extracorporeal life support: a review of the current literature. Eur J Heart Fail. 2017;19(Suppl 2):84-91.

55. Walker CM, Rosado-de-Christenson ML, Martínez-Jiménez S, Kunin JR, Wible BC. Bronchial arteries: anatomy, function, hypertrophy, and anomalies. Radiographics. 2015;35(1):32-49.

56. Wagner EM, Jenkins J, Perino MG, Sukkar A, Mitzner W. Lung and vascular function during chronic severe pulmonary ischemia. J Appl Physiol. 2011; 110(2):538-44

57. Vardi A, Jessen ME, Chao RY, Brink LW, Levin DL, Johnson RL. Effect of extracorporeal membrane oxygenation flow on pulmonary capillary blood flow. Crit Care Med. 1995;23(4):726-32.

58. Al-Ogaili Z, Foulner D, Passage J, Weightman WM, Torre L, Baker S, et al. CT pulmonary angiography during veno-arterial extracorporeal membrane oxygenation in an adult. J Med Imaging Radiat Oncol. 2013;57(3):345-7.

59. Suresh K, Shimoda LA. Lung circulation. Compr Physiol. 2016;6(2):897-943.

60. Schlensak C, Doenst T, Preusser S, Wunderlich M, Kleinschmidt M, Beyersdorf F. Bronchial artery perfusion during cardiopulmonary bypass does not prevent ischemia of the lung in piglets: assessment of bronchial artery blood flow with fluorescent microspheres. Eur J Cardio-Thorac Surg. 2001;19(3):326-31 disciussion 331-332.

61. Koul B, Willen H, Sjöberg T, Wetterberg T, Kugelberg J, Steen S. Pulmonary sequelae of prolonged total venoarterial bypass: evaluation with a new experimental model. Ann Thorac Surg. 1991;51(5):794-9.

62. Koul B, Wollmer P, Willen H, Kugelberg J, Steen S. Venoarterial extracorporeal membrane oxygenation--how safe is it? Evaluation with a new experimental model. J Thorac Cardiovasc Surg. 1992;104(3):579-84.

63. Mizuno T, Tatsumi E, Nishinaka T, Katagiri N, Ohikawa M, Naito H, et al. Observation of alveolar fibrosis in a goat following venoarterial bypass for up to 5 months using extracorporeal membrane oxygenation. J Artif Organs. 2004;7(2):107-9.

64. Ratliff JL, Hill JD, Fallat RJ, Parrot J, Tucker HJ. Complications associated with membrane lung support by venoarterial perfusion. Ann Thorac Surg. 1975; 19(5):537-9.

65. Matthay MA, Zemans RL, Zimmerman GA, Arabi YM, Beitler JR, Mercat A, et al. Acute respiratory distress syndrome. Nat Rev Dis Primer. 2019;5(1):18

66. Schmidt M, Pellegrino V, Combes A, Scheinkestel C, Cooper DJ, Hodgson C. Mechanical ventilation during extracorporeal membrane oxygenation. Crit Care Lond Engl. 2014;18(1):203.

67. Franchineau G, Luyt CE, Combes A, Schmidt M. Ventilator-associated pneumonia in extracorporeal membrane oxygenation-assisted patients. Ann Transl Med. 2018;6(21):427.

68. Bouglé A, Bombled C, Margetis D, Lebreton G, Vidal C, Coroir M, et al. Ventilator-associated pneumonia in patients assisted by veno-arterial extracorporeal membrane oxygenation support: epidemiology and risk factors of treatment failure. PLoS One. 2018;13(4):e0194976.

69. Abdul-Aziz MH, Roberts JA. Antibiotic dosing during extracorporeal membrane oxygenation: does the system matter? Curr Opin Anaesthesiol. 2020;33(1):71-82.

70. Ellouze O, Lamirel J, Perrot J, Missaoui A, Daily T, Aho S, et al. Extubation of patients undergoing extracorporeal life support. A retrospective study. Perfusion. 2019:34(1):50-7.

71. Abrams D, Garan AR, Brodie D. Awake and fully mobile patients on cardiac extracorporeal life support. Ann Cardiothorac Surg. 2019;8(1):44-53.

72. Antoniucci ME, De Paulis S, Bevilacqua F, Calabrese M, Arlotta G, Scapigliati A, et al. Unconventional cannulation strategy in peripheral extracorporeal membrane oxygenation to achieve central perfusion and prevent differential hypoxia. J Cardiothorac Vasc Anesth. 2019;33(5):1367-9.

73. Luedi M, Friess J-O, Erdoes G. Veno-Arterial ECMO Weaning failure in the operating room: have you considered Preweaning bronchoscopy? Artif Organs. 2018;42(12):1234-5.

74. Boulate D, Luyt C-E, Pozzi M, Niculescu M, Combes A, Leprince P, et al. Acute lung injury after mechanical circulatory support implantation in patients on extracorporeal life support: an unrecognized problem. Eur J Cardio-Thorac Surg. 2013;44(3):544-9 discussion 549-550.

75. Asija R, Roth SJ, Hanley FL, Peng L, Liu K, Abbott J, et al. Reperfusion pulmonary edema in children with tetralogy of Fallot, pulmonary atresia, and major aortopulmonary collateral arteries undergoing unifocalization procedures: a pilot study examining potential pathophysiologic mechanisms and clinical significance. J Thorac Cardiovasc Surg. 2014;148(4): 1560-5. 
76. Mayer E, Klepetko W. Techniques and outcomes of pulmonary endarterectomy for chronic thromboembolic pulmonary hypertension. Proc Am Thorac Soc. 2006;3(7):589-93.

77. Weyker PD, Webb CAJ, Kiamanesh D, Flynn BC. Lung ischemia reperfusion injury: a bench-to-bedside review. Semin Cardiothorac Vasc Anesth. 2013; 17(1):28-43.

78. Chen Y-S, Ko W-J, Chi N-H, Wu I-H, Huang S-C, Chen RJ-C, et al. Risk factor screening scale to optimize treatment for potential heart transplant candidates under extracorporeal membrane oxygenation. Am J Transplant. 2004;4(11):1818-25.

79. Combes A, Leprince P, Luyt C-E, Bonnet N, Trouillet J-L, Léger P, et al. Outcomes and long-term quality-of-life of patients supported by extracorporeal membrane oxygenation for refractory cardiogenic shock. Crit Care Med. 2008;36(5):1404-11.

80. Schoenrath F, Hoch D, Maisano F, Starck CT, Seifert B, Wenger U, et al. Survival, quality of life and impact of right heart failure in patients with acute cardiogenic shock treated with ECMO. Heart Lung J Crit Care. 2016; 45(5):409-15.

81. Spangenberg T, Schewel J, Dreher A, Meincke F, Bahlmann E, van der Schalk $\mathrm{H}$, et al. Health related quality of life after extracorporeal cardiopulmonary resuscitation in refractory cardiac arrest. Resuscitation. 2018;127:73-8.

\section{Publisher's Note}

Springer Nature remains neutral with regard to jurisdictional claims in published maps and institutional affiliations. 